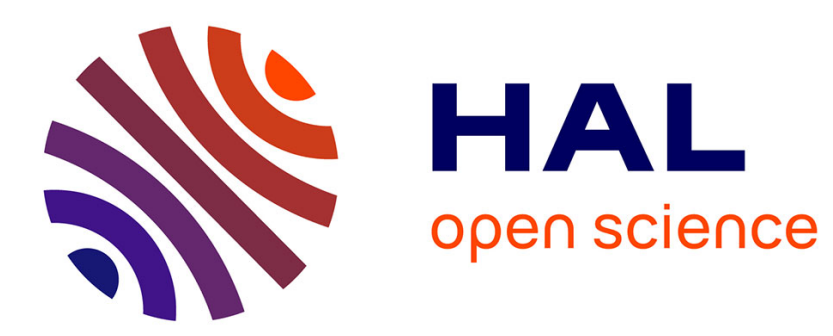

\title{
La distribution du peuplement en Amazonie brésilienne: l'apport des données par secteur de recensement
}

\author{
François-Michel Le Tourneau
}

\section{To cite this version:}

François-Michel Le Tourneau. La distribution du peuplement en Amazonie brésilienne: l'apport des données par secteur de recensement. Espace Géographique, 2009, 2009 (4), pp.359-375. 10.3917/eg.384.0359 . hal-00445755

\section{HAL Id: hal-00445755 \\ https://hal.science/hal-00445755}

Submitted on 10 Nov 2019

HAL is a multi-disciplinary open access archive for the deposit and dissemination of scientific research documents, whether they are published or not. The documents may come from teaching and research institutions in France or abroad, or from public or private research centers.
L'archive ouverte pluridisciplinaire HAL, est destinée au dépôt et à la diffusion de documents scientifiques de niveau recherche, publiés ou non, émanant des établissements d'enseignement et de recherche français ou étrangers, des laboratoires publics ou privés. 


\title{
La distribution du peuplement en Amazonie brésilienne : l'apport des données par secteur de recensement
}

\author{
François-Michel Le Tourneau \\ CNRS - UMR 7169 \\ 28 rue Saint Guillaume 75007 Paris \\ fmlt@,fmlt.net
}

\section{Résumé :}

A partir des données de recensement publiées au niveau des secteurs pour le recensement partiel de 2007, nous proposons ici une approche renouvelée des questions abordées dans un article de 2002 au sujet de l'Amazonie brésilienne, en nous fondant sur une série de questions simples, comme par exemple : «qui vit en forêt ? ", «les populations se concentrent-elles dans les zones défrichées?» ou bien «l'Amazonie est-elle un désert humain?».

L'article propose trois parties. La première est consacrée à la présentation des données, des méthodes de travail et des enjeux liés à la répartition de la population en Amazonie. La seconde partie étudie certaines données du recensement. La troisième partie croise ces données avec d'autres couches d'information géographique, en particulier la carte de la déforestation. En conclusion nous proposons un nouveau découpage régional de l'Amazonie brésilienne.

Mots-clés: Amazonie, SIG, recensement, géographie régionale

\begin{abstract}
:
Census data in Brazil are published at the «block» (the finest) level since 2003, and a new partial census was done in 2007. From these actualised data, we propose here a renewed approach of some points that we presented in a 2002 article about the Brazilian Amazon. The analysis is grounded on a few simple questions such as : "How many people live in the Amazon ?", "How many people do live in the rainforest?" or "Do people in the Amazon live predominantly in deforested areas?".

The present work is divided in three parts. The first presents the data and methods used, as well as the importance of the question we deal with. The second shows some results extracted from the 2007 data. The third crosses data from the census and from other of geographical coverages, in particular the map of the deforested areas of the Amazon. In conclusion, we present a new vision of the regional division of the Brazilian Amazon region.
\end{abstract}

Key-words: Amazon, GIS, census, regional geography

\section{Resumo:}

Usando os dados dos censos populacionais, publicados na escale do setor censitário e os resultados da contagem da população de 2007, apresentamos aqui uma abordagem renovada de problemas relacionados à Amazônia brasileira que já abordamos num trabalho de 2002. A análise fundamenta-se sobre algumas perguntas simples como: "quantas pessoas moram na floresta?", "as populações concentram-se nas áreas desmatadas?" ou "existem áreas vazias na Amazônia?”.

O trabalho é dividido em três partes. A primeira apresenta os dados e os métodos. A segunda analisa os dados da contagem 2007 na Amazônia. A terceira cruza esses dados com outras informações geográficas, em particular o mapa das áreas desmatadas. Para concluir, propomos uma nova divisão geográfica da Amazônia brasileira. 
Palavras chaves : Amazônia, censo demográfico, SIG, geografia regional 


\title{
La distribution du peuplement en Amazonie brésilienne : l'apport des données par secteur de recensement
}

\author{
François-Michel Le Tourneau \\ CNRS - UMR 7169 \\ 28 rue Saint Guillaume 75007 Paris \\ fmlt@,fmlt.net
}

Lors d'un article publié en 2002 (Le Tourneau, 2002), nous nous intéressions aux difficultés de la représentation du peuplement dans le contexte pionnier de l'Amazonie brésilienne, et nous proposions quelques solutions, basées sur des traitements SIG, afin d'y remédier. Depuis le recensement de 2000, publié en 2003, l'Institut brésilien de géographie et statistique, l'IBGE, diffuse les données de recensement au niveau des secteurs ${ }^{1}$, ce qui permet d'améliorer considérablement la vision des phénomènes liés au peuplement, notamment dans les zones rurales.

Fort de ces nouvelles données, nous proposons ici une nouvelle approche des questions abordées précédemment, en nous fondant sur une série de questions simples mais pour lesquelles les réponses sont aujourd'hui beaucoup plus précises, comme par exemple : «qui vit en forêt?", «les populations amazoniennes se concentrent-elles dans les zones défrichées ? » ou bien "l'Amazonie est-elle un désert humain? ». Cette analyse est encore enrichie par le fait qu'un recensement partiel a été effectué en 2007, nous permettant de disposer de données très actualisées.

L'article propose trois parties. La première est consacrée à la présentation des données et des méthodes de travail. Elle souligne également certains des enjeux qui se cachent derrière la question de la répartition de la population en Amazonie. La seconde partie est consacrée à l'étude des données du recensement, en cherchant en particulier à établir quel est le nombre des habitants de la grande forêt amazonienne et comment se répartissent les migrants arrivés depuis dix ans. La troisième partie croise les données du recensement avec d'autres couches d'information géographique, en particulier la carte de la déforestation, afin d'explorer notamment si l'on peut postuler un lien entre la présence d'effectifs de population plus importants et les défrichements.

\section{Problématique et données}

\section{La représentation de la population en Amazonie et ses enjeux}

La représentation de la population à partir de données géoréférencées a déjà montré son potentiel pour l'explication des dynamiques démographiques et territoriales des pays concernés, notamment en Amérique latine (Delaunay, 1994 ; Droulers et al., 2003, Théry et Aparecida de Mello, 2005). Mais dans ce cadre, l'Amazonie pose des problèmes du fait de

\footnotetext{
${ }^{1}$ Les secteurs de recensement sont les unités de collecte des agents recenseurs. Ils se rapprochent des «îlots » définis par l'INSEE en France. On notera que ces données sont vendues par l'IBGE à un coût particulièrement faible, environ $40 €$ pour les 1000 variables statistiques et pour l'intégralité des près de 400000 secteurs de recensement du Brésil. Les mailles géographiques, elles, peuvent être téléchargées gratuitement sur le site web de l'IBGE. Le Brésil, comme d'autres pays latino-américains, suit en cela l'exemple américain de diffusion de l'information statistique et géographique, aujourd'hui rejointe par l'Union européenne dans le cadre de la directive Inspire. Cependant, la diffusion particulièrement ample de données géographiques par les institutions brésiliennes en font plus particulièrement un El Dorado de l'information géographique (Le Tourneau et Théry, 2003).
} 
l'immensité de la région, de la modestie des effectifs de population et de l'hétérogénéité de la maille administrative. Le premier point n'a que peu à être commenté. L'Amazonie brésilienne, définie sur le plan administratif par le concept d'Amazonie légale - sur lequel nous reviendrons - représente 5 millions de $\mathrm{km}^{2}$, soit plus de $61 \%$ de l'ensemble du Brésil et plus de 1 million de $\mathrm{km}^{2}$ de plus que l'Europe des 27. C'est donc un ensemble géographique de première importance. En ce qui concerne le second point, il sera développé en détail dans cet article. La population de l'Amazonie se monte à environ 23 millions d'habitants, ce qui implique des densités de populations faibles dans de nombreuses régions, comme nous le verrons.

Le dernier point, enfin, appelle plus de justifications. Le découpage administratif amazonien, et il est en cela le reflet de celui du Brésil (Théry, Gondard et Velut, 1997), porte la marque du caractère récent de l'effort pionnier et des politiques de développement. Dans les zones affectées, les communes ont été morcelées plusieurs fois (notamment avant 1999, l'opération étant de nos jours plus compliquée à légaliser) et présentent des superficies moyennes qui, si elles sont encore importantes, semblent correspondre à ce que l'on peut imaginer être un territoire municipal. Dans les régions encore isolées, en revanche, les communes sont les héritières des délimitations floues de l'époque coloniale, lors de laquelle on plaçait sous la tutelle d'un chef-lieu récemment créé un immense territoire, pour la plus grande partie inconnu. Les communes géantes, de la taille de certains Etats d'Europe, sont nombreuses. Quatre d'entre-elles dépassent les $100000 \mathrm{~km}^{2}$ : Altamira $\left(160000 \mathrm{~km}^{2}\right)$, Barcelos (123000 km²), São Gabriel da Cachoeira (109 000) et Oriximiná $\left(108000 \mathrm{~km}^{2}\right)$. Dans ces communes, la représentation de la population est problématique car les effectifs concernés sont réduits et le plus souvent concentrés dans une partie très restreinte du territoire concerné, alors que d'immenses zones contiennent des densités extrêmement faibles de populations traditionnelles ou amérindiennes. Dans ce contexte, la publication depuis 2003 des recensements à l'échelle des secteurs apporte une vision indiscutablement plus précise, qui permet de contourner les difficultés que nous soulignions en 2002.

Les enjeux d'une représentation exacte de la localisation de la population de l'Amazonie brésilienne sont nombreux. En premier lieu, la nouvelle vision permet d'établir un diagnostic plus précis de la situation actuelle du peuplement, et de la comparer avec celle, plus médiatisée, du déboisement. Par ce moyen, on peut essayer de comprendre s'il existe un lien immédiat entre la croissance de la population, l'afflux de migrants et la progression des défrichements, ou bien si la relation entre les deux termes est plus subtile, ouvrant la voie à un réflexion sur la question d'un développement durable adapté à la région (Droulers, 2004). Le prisme de la répartition de la population est par ailleurs un facteur explicatif fort des politiques publiques menées, tant au niveau de l'Etat fédéral qu'au niveau des Etats fédérés, dans la région. Comme on le verra, les zones vides d'hommes sont plus souvent l'objet des politiques de préservation, alors qu'elles sont souvent encore isolées et par conséquent peu en danger, alors que les zones les plus peuplées, plus exposées, sont moins souvent prises en compte. C'est donc encore un dilemme auquel doit faire face le gouvernement en Amazonie, pour reprendre l'expression de Fearnside (2003).

Mais l'analyse détaillée de la répartition de la population amazonienne permet aussi d'apporter des éléments dans le débat récurrent sur la nécessité et la justification d'une protection plus stricte de la région. Elle permet en effet de mieux comprendre les conséquences géographiques des positions de ceux qui justifient un usage accru des ressources par les nécessités de la population locale, mais aussi de ceux qui souhaitent limiter la transformation des milieux naturels du fait du rôle de la forêt amazonienne dans les équilibres climatiques continentaux ou dans le cycle du carbone ${ }^{2}$. Enfin, l'étude de la

\footnotetext{
${ }^{2}$ Sur ces deux points, voir Laurance et al., 2001 et Gullison et al., 2007.
} 
répartition de la population permet d'enrichir la vision modélisatrice proposée par certains analystes (voir notamment Soares Filho et al., 2006), en proposant notamment des scénarios sur l'évolution des zones qui demeureraient forestière mais pourraient voir leurs effectifs de population augmenter ou diminuer de manière spectaculaire.

\section{L'apport des données par secteurs du comptage de 2007}

Le Brésil a procédé en 2007 à un dénombrement de la population. Par chance, les critères appliqués pour le choix des municipalités recensées ou non a fait que la région amazonienne a été prise en compte en totalité, à l'exception de quatre communes de l'Etat du Pará (Belém, Ananindeua, Marabá et Santarém), pour lesquelles seules des estimations globales de population ont été publiées. Ce manque n'affecte que peu l'interprétation des données, du moins dans les thématiques que nous souhaitons aborder. Belém et Ananindeua forment en effet une seule et même agglomération dans laquelle la forêt a été défrichée depuis plusieurs siècles. Santarém et Marabá sont des communes de plus petite taille, d'environ 274000 et 197000 habitants, et de superficie un peu plus considérable (22 300 et $\left.15190 \mathrm{~km}^{2}\right)$ mais encore très limitée en comparaison de l'Amazonie entière. Dans les calculs qui suivront, nous considérerons que la population de Belém et celle d'Ananindeua sont à $100 \%$ urbaines. Pour Marabá et Santarém, nous utiliserons le ratio urbain/rural moyen du reste de l'Amazonie pour affecter les effectifs de la population estimée à l'une ou l'autre catégorie (voir tableau 2).

L'intérêt des données 2007 est leur actualité et leur publication au niveau des secteurs, tant sur le plan statistique que sur le plan cartographique. Sur le plan des données chiffrées, on dispose donc d'un univers de 26315 secteurs pour lesquels mille variables sont détaillées, décrivant essentiellement les effectifs de population, la structure par âge, la composition des domiciles et les origines géographiques des personnes recensées. Elles ne prennent pas en compte d'autres variables qui intègrent les recensements complets, comme le niveau d'étude, les revenus, etc. Dans l'idéal, les données du dénombrement pourraient être croisées avec les données plus précises de la PNAD (Pesquisa Nacional por Amostra de Domícilio) pour une analyse multi-niveau, mais celles-ci ne prennent pas en compte les zones rurales des Etats amazoniens, qui sont au cœur de l'analyse que nous présentons ici. La qualité des données de l'IBGE est reconnue, et les agents qui ont travaillé au dénombrement 2007 ont été équipés de PDA incorporant un GPS et un logiciel ad hoc de collecte des données, si bien que la qualité globale des données analysées peut être considérée comme bonne, représentant en tout cas de manière fidèle les changements en cours.

Au niveau cartographique, la maille des secteurs publiée par l'IBGE pour l'ensemble de la région comprend 13736 unités, soit une vision 17 fois plus détaillée que celle des 756 communes le plus souvent utilisée. Certes les disparités entre secteurs de grande taille (et il y en a, un secteur de la commune d'Oriximiná représentant à lui seul près de $63000 \mathrm{~km}^{2}$ ) et secteurs plus restreints existent encore. Comme le souligne la documentation de ces données $^{3}$, les secteurs de recensement sont avant tout le produit d'une grille de répartition du travail entre les agents effectuant le recensement, ce qui signifie que leur répartition de l'espace, même si elle obéit dans ses grandes lignes à la division politico-administrative du pays, n'est pas significative en soi. Bien que leur découpage doive au départ permettre une homogénéité en volume (chaque secteur doit représenter environ 205 domiciles, soit 1000 personnes) et en superficie (un secteur doit pouvoir être parcouru pas un agent de recensement), ces critères sont absolument impossibles à respecter en dehors des zones

\footnotetext{
${ }^{3}$ «Le secteur censitaire est une unité territoriale créée afin de collecter et de contrôler les données. Les secteurs ont des limites physiques identifiables sur le terrain, qui respectent les limites politico-administratives légales des zones rurales, urbaines ou autres. Les secteurs comportent un nombre de domicile adéquat pour les opérations de recensement. [...] » (IBGE, 2008 :3)
} 
urbaines, en particulier en Amazonie, pour des raisons de dispersion de la population. Pour autant, la multiplication des unités spatiales permet de mitiger les effets de l'hétérogénéité en taille des secteurs, puisque la plus grande partie des effectifs de population est groupée dans des secteurs de petite taille, représentant le plus souvent des villes ou des villages, alors que les effectifs de population dispersés sur les secteurs de grande taille sont cette fois véritablement très réduits (à la différence de l'échelle municipale, à laquelle on diluait l'ensemble de la population locale sur la superficie, parfois immense, de la commune).

Comme on l'aura noté, le nombre des secteurs de la maille est inférieur de moitié à celui des statistiques. En effet, les maille publiée à l'échelle du Brésil entier ne détaille pas les secteurs (extrêmement nombreux et de très petite taille) à l'intérieur des agglomérations urbaines. En remplacement, elle comprend un polygone qui est la réunion des secteurs urbains considérés. Le premier traitement géographique que nous avons effectué a donc consisté à affecter à ces secteurs urbains la somme de la population des secteurs qu'ils contenaient.

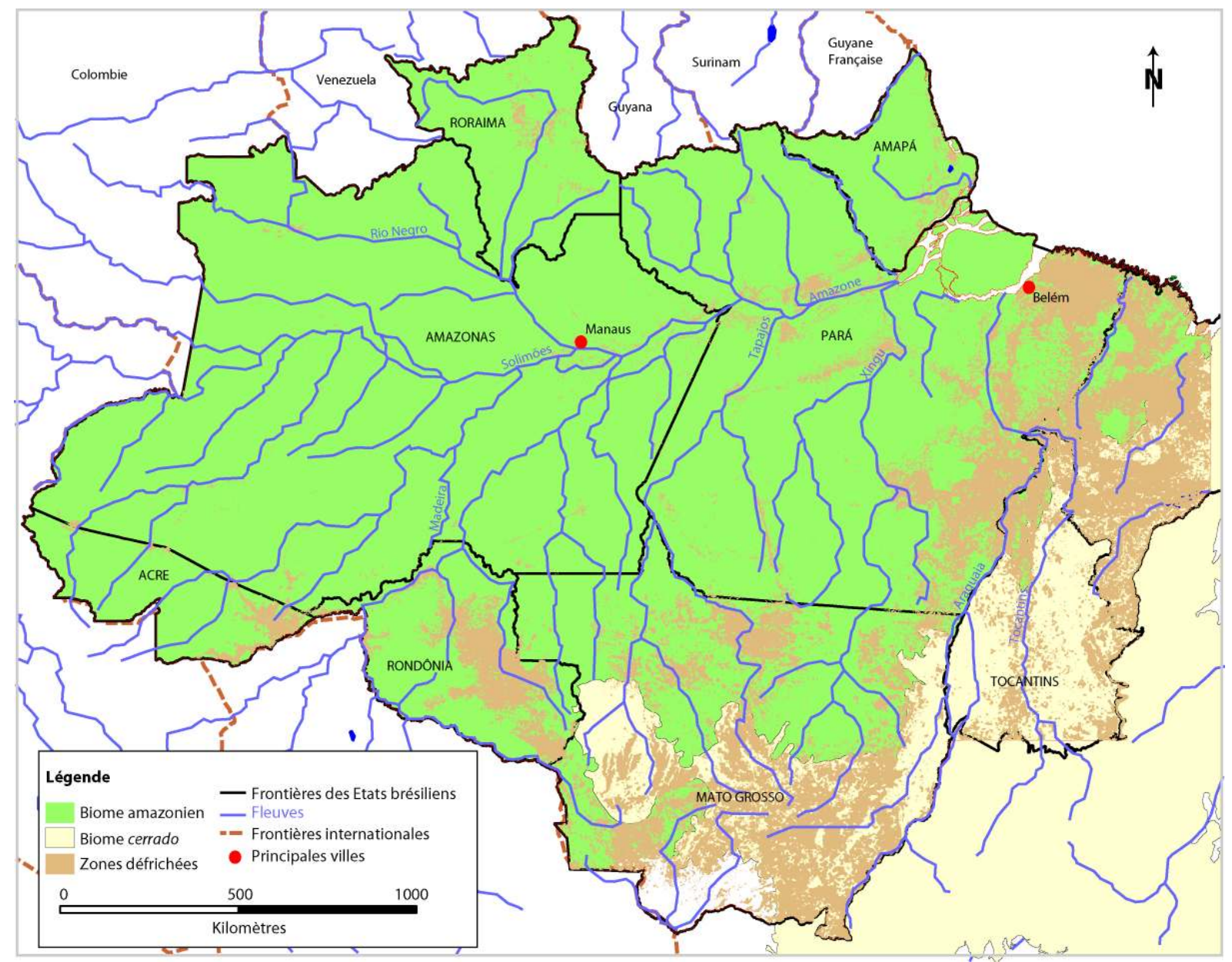

Carte 1 : la région d'étude.

\section{Croisements avec d'autres données}

La puissance des systèmes d'information géographique réside dans leur capacité à croiser des données spatiales de sources et de nature différentes. Pour cette étude, nous utilisons plusieurs données en plus de la maille des secteurs de recensement. 
La première est une cartographie des biomes du Brésil diffusée par le ministère brésilien de l'environnement ${ }^{4}$ (voir Carte 1), qui découpe le territoire national en six principales régions écologiques. La région amazonienne y est limitée à l'écosystème de la forêt tropicale (même si elle inclut un certain nombre de sous-régions de faciès différent) et s'étend sur l'ensemble des zones recouvertes de forêt tropicales avant l'apparition de la déforestation à grande échelle. Au total, ce domaine recouvre 4,2 millions de kilomètres carrés.

Nous utilisons également la cartographie de la déforestation publiée dans le cadre du projet PRODES numérique par l'INPE (Institut brésilien d'études spatiales) ${ }^{5}$. Ces données sont divulguées à l'échelle de l'Amazonie entière sous la forme d'une image matricielle (raster) classifiée avec une résolution de 60 mètres par pixel. Nous avons converti les différentes classes de déforestation en données vecteur afin de pouvoir les croiser avec la maille des secteurs de recensement. Les données de déforestation produites par l'INPE sont les données de référence sur le sujet (avec un total de zones défrichées de $691284 \mathrm{~km}^{2}$ en 2007), mais elles possèdent un certain nombre de biais qui doivent être pris en compte lors des interprétations. Le plus important est l'existence de petites zones peu ou pas cartographiées du fait de leur ennuagement ou de leur très faible déforestation. Afin de limiter les effets de ces problèmes, nous avons complété la base de données de 2007 par des données plus anciennes, également produites par l'INPE.

Nous utilisons également une couche comprenant l'ensemble des aires protégées de 1'Amazonie : terres indigènes, parcs nationaux, réserves extractivistes, forêts nationales, etc.. Cette couche a été confectionnées par nos soins à partir de la compilation de données provenant des institutions responsables de ces aires protégées (FUNAI, IBAMA, etc.) mais aussi de certaines ONG qui mènent une veille sur ce sujet.

Nous utilisons enfin une série de données sur le zonage global de l'Amazonie brésilienne et sur la maille routière, prises directement sur les serveurs de données géographiques du ministère de l'environnement et du programme SIPAM ${ }^{6}$.

\section{Méthodes et outils}

Le traitement des données a été effectué principalement avec deux logiciels, Mapinfo (et son extension Mapbasic) et Envi. Les données vecteur (maille des secteurs de recensement et couches vecteurs sur l'Amazonie légale et sur les écosystèmes) ont été converties du format shape vers le format de Mapinfo et utilisées par la suite dans ce logiciel. Les données de déforestation ont été généralisées et vectorisées dans le logiciel Envi avant d'être exportées en format vecteur vers Mapinfo.

Le traitement des données statistiques afin de renseigner les effectifs de population de chaque secteur de recensement (en additionnant les données pour les secteurs urbains) a été effectué grâce à des routines Mapbasic programmées par l'auteur. La plupart des calculs présentés ici a été effectuée en utilisant les fonctionnalités SQL de Mapinfo, qui permettent de combiner des requêtes classiques de bases de données avec des opérateurs géographiques (objet contenu / contenant, intersection, distance, etc.). Pour le calcul de la proportion de déforestation de chacun des secteurs de recensement, la cartographie de la déforestation a été découpée en fonction de la maille des secteurs, puis la superficie des objets contenus dans chacun des secteurs ajoutée et comparée à la superficie totale du secteur.

\footnotetext{
${ }^{4}$ Cartographie produite en coopération par le Ministère de l'environnement (MMA) et l'Institut brésilien de géographie et statistique (IBGE). Données disponibles sur http://mapas.mma.gov.br/i3geo/datadownload.htm

${ }^{5}$ Voir http://www.obt.inpe.br/prodes/index.html

${ }^{6}$ Système de Protection de l'Amazonie. Ce grand programme, dénommé SIVAM à l'origine, propose entre ses nombreuses activités un service de partage de données géographiques sur l'Amazonie.
} 


\section{La répartition de la population en Amazonie brésilienne}

\section{Combien de personnes vivent-elles en Amazonie?}

Le Brésil dispose d'une délimitation administrative de la région amazonienne, que l'on appelle «Amazonie légale ». Celle-ci ne correspond pas exactement à d'autres découpages administratifs, puisque si huit Etats sont intégralement compris dans l'Amazonie légale (Acre, Amapá, Amazonas, Mato Grosso, Pará, Rondônia, Roraima), le Maranhão ne l'est que partiellement (à l'ouest du méridien $45^{\circ} \mathrm{W}$, voir Carte 1). Par ailleurs, les neuf Etats d'Amazonie légale appartiennent à trois régions administratives, la région Nord (Acre, Amapá, Amazonas, Pará, Rondônia, Roraima), la région Nordeste (Maranhão) et la région Centroeste (Mato Grosso). De ce fait, la détermination de la population de l'Amazonie légale ne peut se faire simplement par l'addition des totaux de population des Etats. En utilisant un croisement SIG, on parvient cependant rapidement à établir le total de la population des secteurs compris dans cette zone, soit 19,945 millions d'habitants. Il faut leur ajouter l'estimation de 2,363 millions de personnes vivant dans les quatre communes qui n'ont pas été incluses dans le recensement. La population de l'Amazonie légale s'établit donc à 22,308 millions d'habitants.

On ne saurait néanmoins en conclure que l'ensemble de ces personnes vivent dans un écosystème forestier (ou qui fut forestier). En effet, lors de la délimitation du périmètre de l'Amazonie légale ${ }^{7}$ on a taillé large, et ajouté aux régions forestières d'autres zones que l'on souhaitait également voir bénéficier des nouveaux mécanismes de subvention. Environ un million de kilomètres carrés de savanes du plateau central brésilien a ainsi été inclus dans l'Amazonie légale. Or, c'est principalement dans ces savanes et à leur interface que s'est développé la grande agriculture qui fait aujourd'hui débat en Amazonie (Bertrand et al., 2003 ; Dubreuil et al., 2007). Ces zones ont également vu une croissance très importante de leur population. Il paraît donc important de pouvoir distinguer les deux aires, l'Amazonie forestière proprement dite et sa bordure sud composée de cerrados (Carte 1 et 2). Nous utilisons pour ce faire la cartographie des milieux naturels brésiliens présentée en I.3.

\footnotetext{
${ }^{7}$ Loi 1.806, du 6 janvier 1953, article 2, reprise par la loi no 5.173 du 27 octobre 1966, article 2, puis modifiée en 1977 et en 1988 lors de la création des Etats du Mato Grosso do sul et du Tocantins.
} 


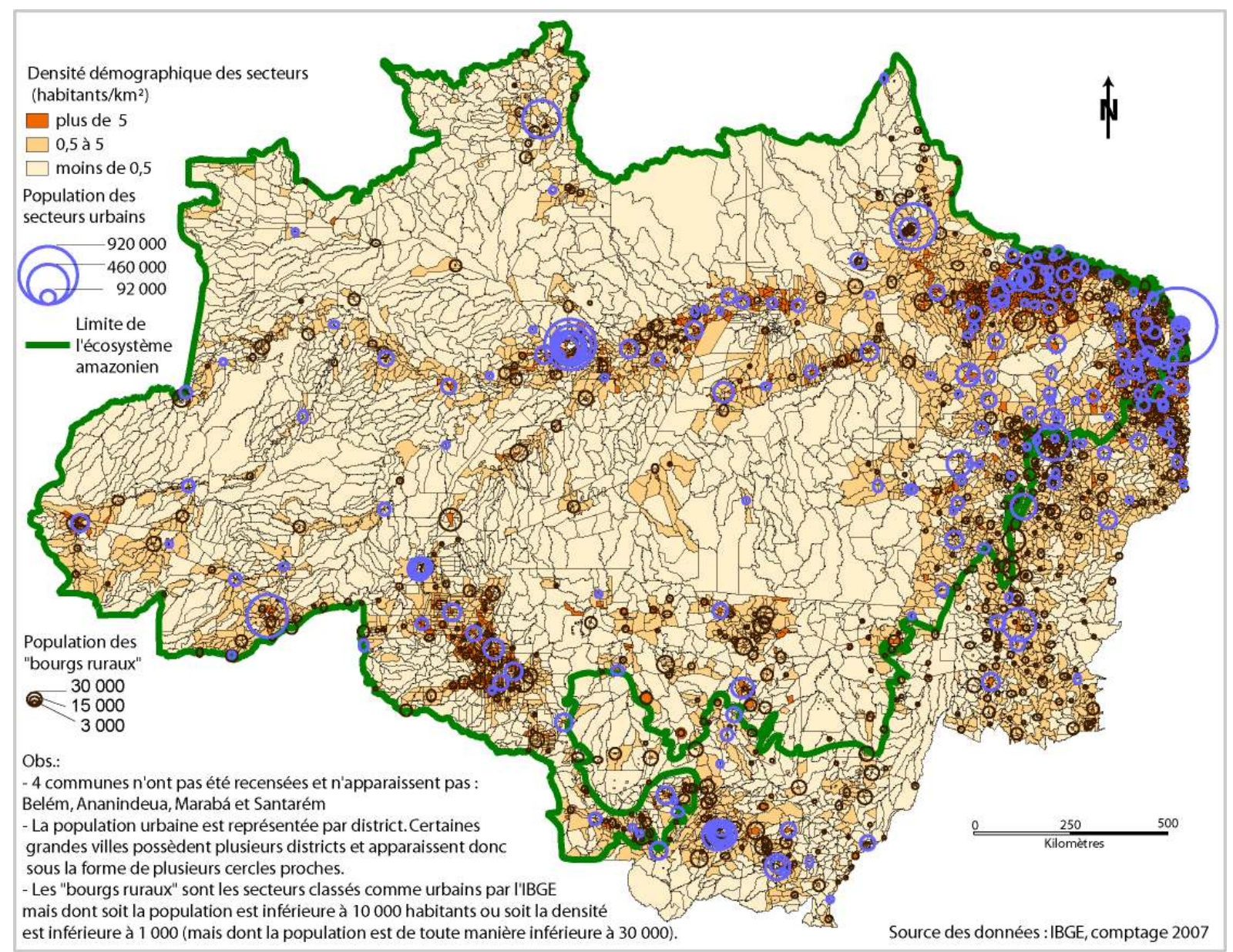

Carte 2 : Localisation de la population amazonienne par secteur de recensement

Selon ce nouveau calcul, la population amazonienne proprement dite n'est plus que de 17,906 millions - un chiffre éloigné des 25 millions souvent avancés par le gouvernement brésilien $^{8}$. Les savanes du sud rassemblent, pour leur part, 4,435 millions d'habitants.

\section{Redéfinir population urbaine et population rurale?}

Si l'on considère donc que presque 18 millions d'habitants demeurent en Amazonie, peuton pour autant les considérer comme des habitants de la forêt? A l'évidence, non, puisqu'une partie importante de ces personnes habitent dans un milieu urbain, qui n'entretient que de lointains rapports avec l'écosystème qui l'entoure. Il nous faut donc nous interroger sur la répartition entre population urbaine et population rurale dans la région amazonienne.

\begin{tabular}{|l|r|l|r|r|r|r|}
\hline Situation & $\begin{array}{l}\text { Population } \\
\text { totale } \\
\text { (millions) }\end{array}$ & $\begin{array}{l}\text { Population } \\
\text { des } \\
\text { secteurs } \\
\text { non } \\
\text { recensés }\end{array}$ & Total & Pourcentage & $\begin{array}{l}\text { Superficie totale } \\
\left(\text { milliers de } \mathrm{km}^{2}\right)\end{array}$ & $\begin{array}{l}\text { Densité } \\
\text { moyenne }\end{array}$ \\
\hline 1 (Urbain) & 10,399 & 2,245 & 12,644 & 70,61 & 11,396 & 1109,51 \\
\hline 2 & 0,027 & & 0,027 & 0,15 & 0,579 & 46,63 \\
\hline
\end{tabular}

\footnotetext{
${ }^{8}$ Voir par exemple les propos du Ministre des affaires stratégiques Mangabeira Unger lors de la session extraordinaire du 21/05/2008 de la commission de l'Amazonie et de l'intégration nationale et de la commission de l'environnement et du développement durable de la chambre des députés.
} 


\begin{tabular}{|l|r|r|r|r|r|r|}
\hline $3 \quad 0,006$ & & 0,006 & 0,03 & 0,001 & 6000,00 \\
\hline $\begin{array}{l}\text { (Petites } \\
\text { villes) }\end{array}$ & 0,212 & & 0,212 & 1,18 & 0,107 & 1981,31 \\
\hline $\begin{array}{l}5 \quad \text { (Bourgs } \\
\text { ruraux) }\end{array}$ & 0,982 & & 0,982 & 5,48 & 7,950 & 123,52 \\
\hline 6 & 0,023 & & 0,023 & 0,13 & 0,153 & 150,33 \\
\hline 7 & 0,125 & & 0,125 & 0,70 & 60,463 & 2,07 \\
\hline 8 (Rural) & 3,732 & 0,155 & 3,887 & 21,71 & 4142,62 & 0,94 \\
\hline TOTAL & 15,506 & 2,4 & 17,906 & 100 & 4223,26 & \\
\hline
\end{tabular}

Tableau 1 : population et surface cumulées des secteurs de recensement selon leur situation (données IBGE, comptage 2007).

L'organisme qui effectue le recensement, l'IBGE, classe les secteurs sur une échelle allant de l'urbain au rural, en passant par les petites villes ou les bourgs ruraux. Selon cette classification (tableau 1), près de $71 \%$ de la population amazonienne habitent dans des secteurs urbains. Ce chiffre confirmerait l'expression de la géographe Bertha Becker, qui décrit l'Amazonie comme "une forêt urbanisée » (Becker, 2004) et l'amplifierait même, puisque le taux d'urbanisation n'était que de $68 \%$ en 2000.

Mais la classification urbaine ou rurale des secteurs a fait l'objet d'un intense débat après la publication des résultats du recensement de 2000. José Eli da Veiga (2002) dénonça par exemple les villes "imaginaires » créées par la classification de l'IBGE en montrant que nombre des secteurs dits « urbains » ne sont en fait classés dans cette catégorie que du fait de leur catégorie administrative et non en raison de leurs fonctions, de leurs équipements ou de leur place dans les flux régionaux. Les données IBGE minimiseraient donc le monde rural brésilien, pourtant encore particulièrement fort et présent dans la vie du pays. Au vu de ce débat, il est tentant de creuser un peu plus la question en ce qui concerne l'Amazonie. Si l'on considère que la notion d'urbanité implique, en premier lieu, un nombre relativement important de personnes concentrées dans une surface relativement petite ${ }^{9}$, il est possible d'utiliser les capacités de calcul géographique de notre SIG pour retravailler ces chiffres selon une approche binaire urbain rurale qui peut paraitre moins fine que l'approche par gradient en principe utilisée par l'IBGE (tableau 1), mais disqualifiée par la sur-représentation des deux catégories extrêmes : en l'absence d'une limite claire entre urbain et rural, comment édicter des sous-catégories?

Seuls 10,5 millions de personnes environ ${ }^{10}$ habitent dans des secteurs qui ont soit à la fois une population supérieure à 10000 habitants et une densité supérieure à 1000 habitants par kilomètres carrés, soit une population supérieure à 30000 personnes. Nous considérons ces caractéristiques comme le seuil d'entrée dans le monde urbain puisqu'elles supposent à la fois un habitat dense et des effectifs de population justifiant des équipements urbains (hôpital, etc.). Elles sont une tentative de transposition de notre part des seuils avancés dans Veiga (2004), mais qui se situaient au niveau des communes et se trouvaient donc dilués par rapport aux données par secteur.

Le résultat est présenté en cercles proportionnels bleus sur la carte 2 . « Seuls » $58 \%$ de la population amazonienne se trouveraient dans ce cas. La différence de près de $13 \%$ avec l'évaluation précédente provient certainement de nombreux petits bourgs ou chefs-lieux qui jouent un rôle administratif mais ne possèdent que de faibles effectifs de population, habitant un tissu encore peu dense (représentés en cercles proportionnels marrons, avec une échelle

\footnotetext{
${ }^{9}$ Les spécialistes de géographie urbaine nous excuserons une définition aussi pauvre mais exploitable dans le cadre de notre SIG.

${ }^{10}$ En tenant compte de la population des communes non-recensées, voir I.2.
} 
différente, sur la carte 1). Ces bourgs ou villages sont totalement dépendants de l'activité agricole ou extractiviste et peuvent être considérés comme participant au monde rural.

De manière intéressante, la différence entre l'urbanisation signalée par l'IBGE et celle que nous calculons est encore plus grande pour la partie non-forestière de l'Amazonie légale. Environ $75 \%$ de la population (3,341 millions d'habitants) y résident en effet dans des secteurs «urbains» selon l'IBGE, contre moins de $51 \%$ (2,273 millions) selon notre définition. On peut voir dans ce chiffre la confirmation de notre interprétation ci-dessus. Dans ces régions, le plus souvent occupées par de la grande agriculture, de petits bourgs d'appui aux activités agricoles se sont développés, demeurant de petite taille et peu urbanisés, au détriment du développement de plus grandes agglomérations (limitées à une quinzaine de villes). La carte 1 montre clairement ce phénomène.

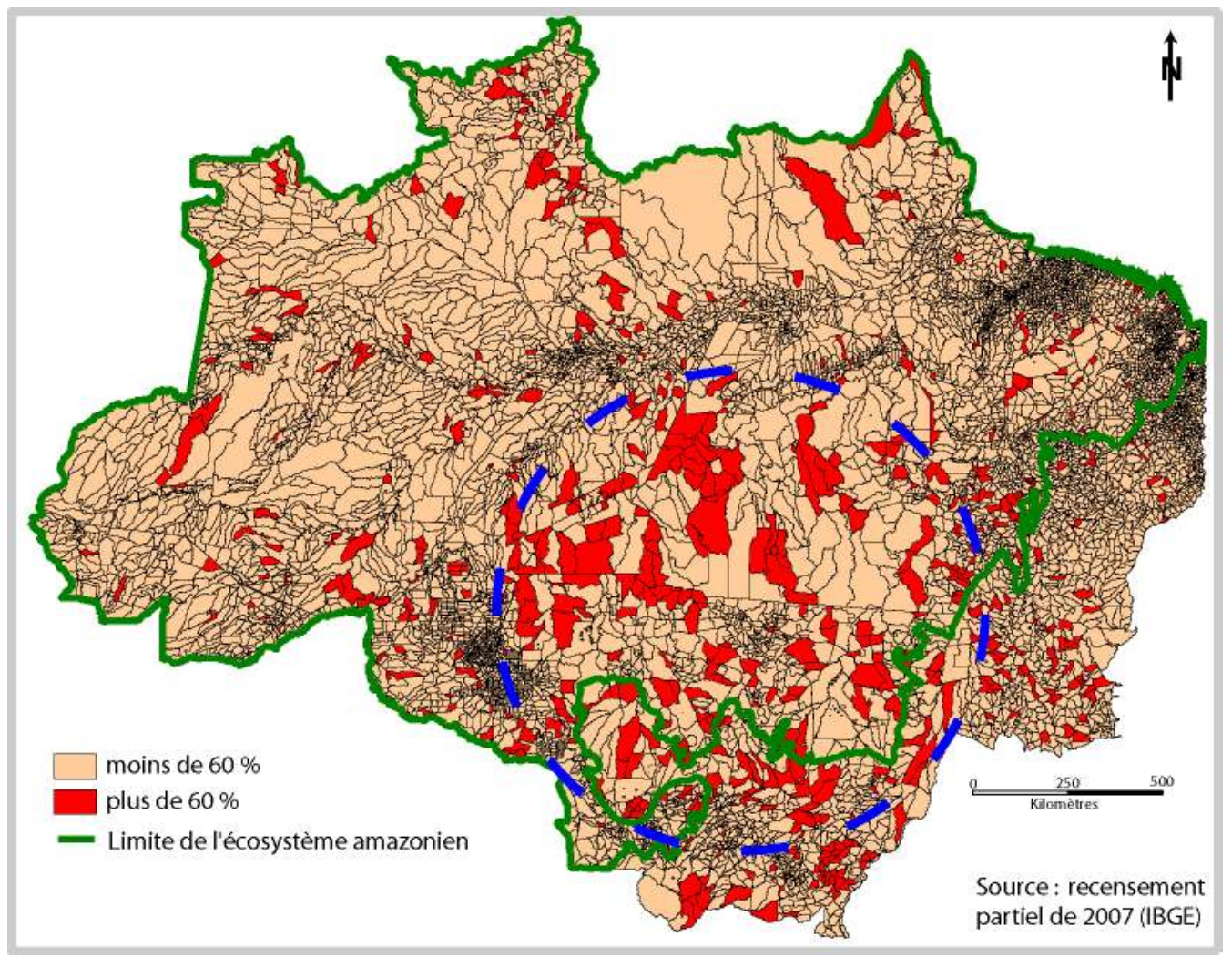

Carte 3 : rapport de masculinité dans les secteurs ruraux en Amazonie.

\section{Les migrations}

Les données du comptage 2007 proposent des informations sur les déplacements de population. Elles distinguent en effet les personnes qui résident depuis avant l'année 2000 dans la même commune et celles qui sont arrivées plus récemment. Pour ces dernières, on sait si elles proviennent d'une autre commune du même Etat ou bien d'un autre Etat (ou bien de l'étranger).

L'analyse globale de ces chiffres est déjà significative. En Amazonie légale, un peu plus de 1,1 millions de personnes (sur 22,3) se sont déplacées au sein du même Etat, alors que 0,7 millions de personnes se sont installées en Amazonie depuis 2000, dont seulement 356000 en provenance d'un Etat extérieur à l'Amazonie légale ${ }^{11}$. On assiste en somme à un

\footnotetext{
${ }^{11}$ Cette distinction est importante car les effets de frontière entre les Etats amazoniens ont parfois prêté à confusion. On a ainsi en 2000 beaucoup montré l'Amapá comme une nouvelle terre d'immigration en Amazonie, du fait d'un nombre de migrants très importants venus d'autres Etats. Mais la plus grande partie de ceux-ci
} 
ralentissement important de l'immigration depuis l'extérieur (le nombre total de personnes provenant des Etats non amazoniens approchait les 800000 personnes pour la période 19902000). Souvent désignée en Amazonie comme « envahissante », on notera que la migration en provenance de l'Etat du Maranhão semble aussi diminuer. Seuls 112000 personnes de cet Etat se sont installées en Amazonie durant la période considérée, soit tout de même encore $15 \%$ des effectifs totaux des migrants. Les mouvements internes à l'Amazonie, diminuent eux aussi à peu près dans les mêmes proportions. En revanche, l'instabilité au sein des Etats demeure forte.

L'analyse par secteurs permet de mieux cerner les destinations favorites des migrants et en particulier de continuer à travailler sur la question urbain/rural abordée au point précédent. Or si l'on reprend les secteurs urbains (selon notre définition), on constate que ceux-ci ont attiré un peu plus de $61 \%$ de l'ensemble des migrants. La distribution des migrants semble donc rejoindre la configuration régionale, sans que l'on ne puisse distinguer une attirance plus forte soit pour les secteurs urbains ou soit pour les secteurs ruraux. Les migrants qui se déplacent au sein du même Etat semble, eux, privilégier les zones rurales puisqu'ils se répartissent à peu près à égalité entre secteurs urbains et secteurs ruraux. Si l'on distingue l'immigration vers les zones forestières et celle vers les régions de cerrado, on note une différence plus importante. Les secteurs participants du biome forestier attirent en effet $70 \%$ du total des migrants (soit presque 500000 personnes), et plus de $80 \%$ des migrants provenant d'une autre région du pays. Ceux-ci se dirigent à $65 \%$ vers les secteurs urbains. Si l'on considère les migrants qui ont pour destination un secteur rural, environ un tiers ont choisi de s'installer dans le cerrado, ce qui semble indiquer une certaine prééminence de ce dernier vu qu'il ne représente que $17 \%$ de la surface totale de l'Amazonie légale.

\section{La marque du front pionnier...}

Le rapport de masculinité permet de caractériser plusieurs régions au Brésil (Théry et de Mello, 2004). Lorsque la population féminine est largement supérieure à la population masculine, on est en présence de régions d'émigration, dans lesquelles la main d'œuvre jeune et souvent masculine est partie vers des régions plus attractives (cas du Nordeste), ou bien de régions métropolitaines dans lesquelles les emplois domestiques ont attiré une proportion importante de population féminine. Lorsque la population masculine l'emporte nettement, on est en présence de zones pionnières dans lesquelles le type de travail proposé (bûcheronnage, orpaillage, défrichements, emplois de cow-boys, agriculture pionnière) est le plus souvent entrepris par des hommes, qui sont donc attirés dans ces zones par les offres d'emploi. C'est le cas de certaines régions de l'Amazonie.

La cartographie du rapport de masculinité par secteur de recensement permet d'affiner la vision de ce phénomène. On note ainsi dans la carte 3 que les secteurs ruraux présentant une forte proportion d'hommes sont particulièrement nombreux dans le sud de l'Amazonie. Ils ne semblent pas configurer un front linéaire, mais bien plus une zone circulaire (matérialisée en bleu sur la carte) au sein de laquelle de profondes transformation du territoire sont en cours. Et, de fait, cette région sud de l'Amazonie, bordée au nord par la Transamazonienne et le Rio Madeira et au sud par la transition avec les savanes du plateau central brésilien, est la région dans laquelle se concentrent les ingrédients du front pionnier actuel : exploitation forestière, avancée de l'élevage bovin, extension des grandes cultures et violence rurale. Il est intéressant de noter que de nombreux secteurs qui présentent une forte dissymétrie entre population masculine et population féminine sont situés en avant des fronts de déforestation actuels. Ils

provenaient en fait de zones de l'Etat voisin du Pará pour qui Macapá, capitale de l'Amapá est le centre habituel de services. Il s'agissait donc d'une migration classique vers une grande ville depuis les zones rurales polarisées par elle et non d'un nouveau flux en direction de l'Amazonie. 
sont sans doute autant de marqueurs de la situation à venir, et pourraient à cet égard être utilisés pour enrichir les modèles de prévision de la déforestation (Voir Soares Filho et al., 2006).

\section{Population et déforestation}

\section{La population des zones défrichées}

L'usage du SIG nous permet de tenter de voir si la population qui habite l'écosystème amazonien occupe des secteurs dans lesquels la déforestation a été intense ou non (tableau 2 et carte 3$)^{12}$. Nous nous restreignons ici aux 7,276 millions de personnes occupant des secteurs ruraux (selon notre définition) dans la mesure où il est clair que les secteurs urbains sont, par définition, des secteurs défrichés.

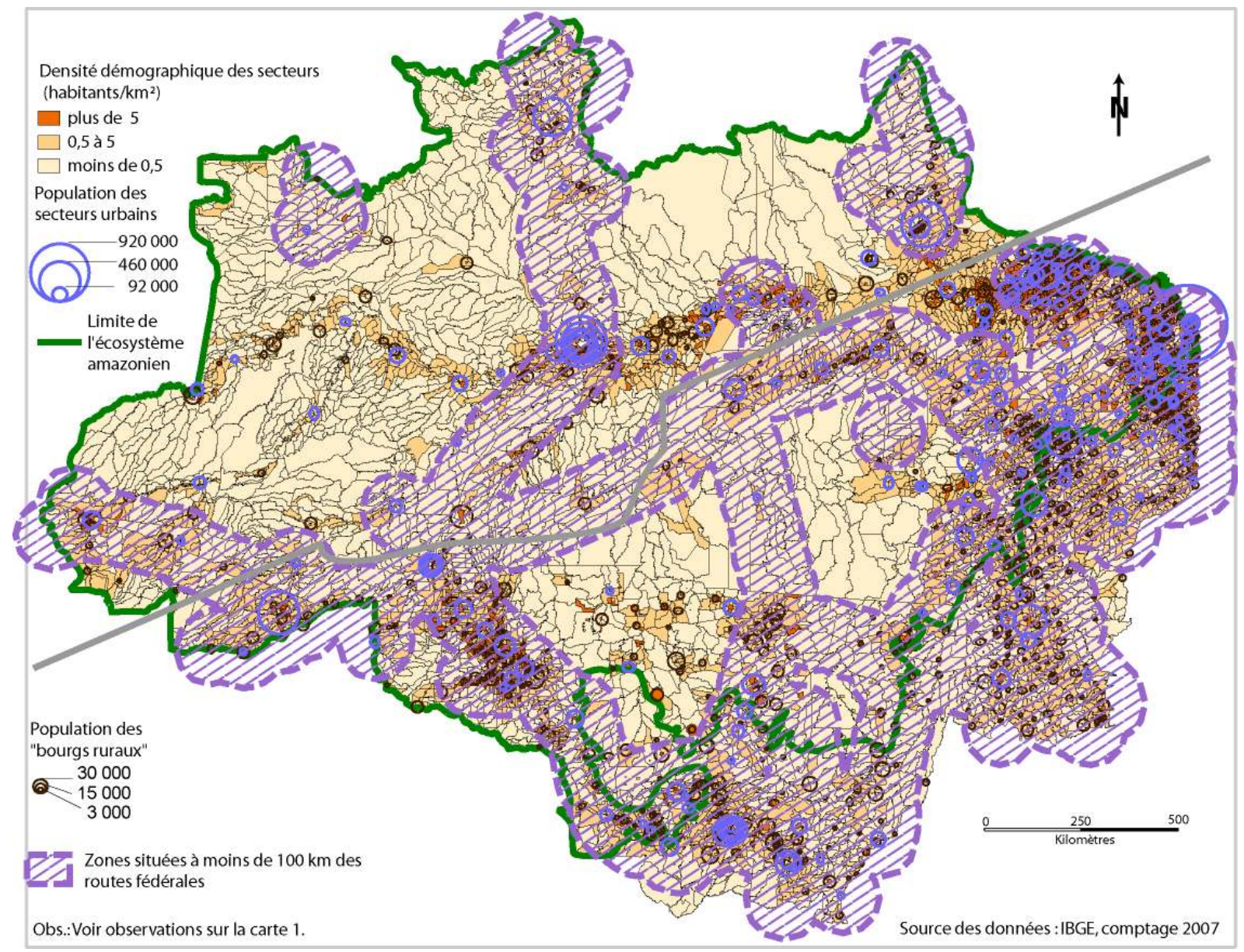

Carte 4 : densité des secteurs de recensement, population des bourgs urbains et distance aux routes fédérales.

Le résultat du croisement nous montre que 59,6 \% de la population considérée habite dans des secteurs donc la proportion de déforestation est supérieure à la moitié. L'ensemble de ces secteurs représente 502000 kilomètres carrés, ou $12 \%$ de l'écosystème amazonien. Cette population importante (4,3 millions) et sa relative densité (environ 8 habitants par $\mathrm{km}^{2}$ ) est le

\footnotetext{
${ }^{12}$ Cette évaluation ne peut se réaliser que dans les zones correspondant à l'écosystème amazonien car le programme PRODES ne réalise pas d'évaluation des transformations de l'écosystème du cerrado.
} 
résultat des politiques de colonisation menées depuis les années 1970 (et toujours d'actualité, voir Arnauld de Sartre, 2006).

Comme on l'avait observé en 2002 à la suite de Hervé Théry, on peut observer une relation étroite entre la présence de cette population rurale et la maille des routes fédérales établie à partir des années 1960. Si l'on considère l'espace situé à moins de 100 kilomètres de ces axes de transport (espace que la dictature militaire avait placé sous sa domination directe afin, justement, d'en assurer le «développement»), on constate (Carte 4) qu'il recouvre presque en totalité les secteurs les plus denses, près de 78,5\% de la population rurale (selon notre définition) amazonienne se concentrant dans des secteurs entièrement inclus dans ce « tampon », et qu'il inclut tous les noyaux urbains importants. Ces secteurs rassemblent par ailleurs plus de $75 \%$ de la déforestation totale, ce qui explique pourquoi la construction des routes, ou leur amélioration, est souvent considérée dans les modèles prédictifs comme entraînant mécaniquement de la déforestation. Or si l'on observe dans le détail la carte, on se rend compte qu'il existe dans cette relation une dissymétrie dans la mesure où au sud de la ligne grise indiquée sur la carte la relation est presque parfaite tandis qu'au nord, l'espace des routes semble croiser de manière plus aléatoire l'espace peuplé, laissant en particulier toute la vallée de l'Amazone et celles de ses principaux affluents en dehors. L'opposition classique entre Amazonie des fleuves et Amazonie des routes semble donc encore d'actualité aujourd'hui, alors que par ailleurs il semble falloir en conclure que la simple existence d'un axe routier ne suffit pas à le transformer en vecteur de peuplement ou de déforestation, encore faut-il aussi qu'il existe également un lien avec le sud du pays.

La relation entre la présence de population et la déforestation n'est pas non plus si claire. Une bonne proportion de la population rurale amazonienne (environ $30 \%$ ) réside dans des secteurs peu défrichés (moins de $25 \%$ ). Presque $16 \%$ (1,148 millions d'habitants) habitent même dans des régions particulièrement peu touchées par la déforestation puisqu'elle y est inférieure à $1 \%$. Contrairement à ce que l'on pourrait penser, il ne s'agit pas de populations amérindiennes, car, d'abord, leur nombre est inférieur en Amazonie à 300000 personnes et car, ensuite, il est bien connu que le recensement de l'IBGE prend particulièrement mal en compte ce type de population. On peut donc considérer qu'il existe au moins 1,15 millions de personnes habitant les zones forestières tout en maintenant un très faible impact sur leur environnement. Ce dernier point ne paraîtra pas étonnant si l'on rapporte cette population à l'immensité de l'espace considéré (près de 2,3 millions de kilomètres carrés). La très faible densité de population expliquerait la faiblesse de l'impact. 


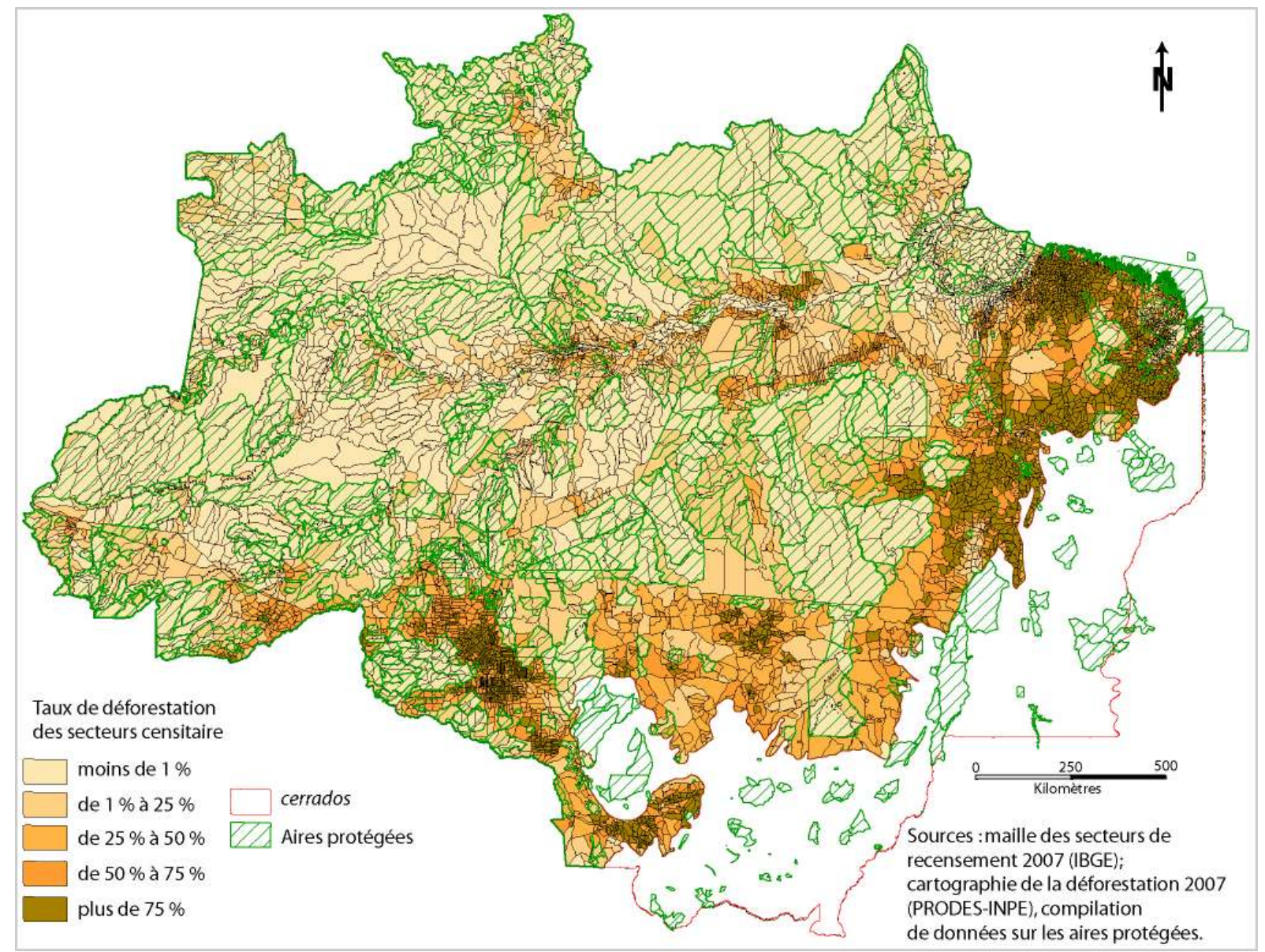

Carte 5 : Taux de déforestation des secteurs de recensement.

\begin{tabular}{|l|r|r|r|}
\hline $\begin{array}{l}\text { Pourcentage de deforestation } \\
\text { dans le secteur }\end{array}$ & $\begin{array}{l}\text { Population } \\
\text { totale (millions) }\end{array}$ & $\begin{array}{l}\text { Superficie totale } \\
\left(\text { millions } \mathrm{km}^{2}\right)\end{array}$ & $\begin{array}{l}\text { Densité } \\
\text { moyenne }\end{array}$ \\
\hline Moins de $1 \%$ & 1,148 & 2,292 & 0,50 \\
\hline 1 to $25 \%$ & 1,036 & 1,045 & 0,99 \\
\hline 25 to $50 \%$ & 0,752 & 0,378 & 1,98 \\
\hline $50 \%$ to $75 \%$ & 1,015 & 0,243 & 4,17 \\
\hline Plus de $75 \%$ & 3,323 & 0,259 & 12,83 \\
\hline
\end{tabular}

Tableau 2 : population rurale des secteurs de recensement en fonction de leur taux de déforestation.

\section{Le rôle des migrations}

La conversion des forêts amazoniennes en terres agricoles a souvent été associée à un mouvement migratoire en direction des zones rurales amazoniennes, ainsi que la propagande de l'époque militaire souhaitait le faire entendre ${ }^{13}$. Comme on l'a vu au paragraphe précédent, cette vision n'est pas totalement erronée, au vu du nombre d'habitants des zones défrichées. Mais ce raisonnement est-il encore valable aujourd'hui?

En premier lieu, on doit noter la faiblesse actuelle des effectifs en jeu. Seules 179000 personnes ont immigré dans un secteur rural participant du biome forestier, ce qui paraît peu en regard des décennies précédentes. Par ailleurs, sur ce total, 102000 personnes provenaient

\footnotetext{
${ }^{13}$ Selon la formule consacrée les militaires proposaient de donner « les terres sans homme d'Amazonie aux hommes sans terre du Nordeste $»$.
} 
déjà d'un Etat amazonien ${ }^{14}$, si bien que la migration depuis une autre région du Brésil en direction des zones rurales forestières se limite à environ 77000 personnes. Beaucoup plus significatifs sont les effectifs de personnes qui ont changé de commune dans le même Etat, 434000 d'entre eux s'étant établis en zone rurale et en forêt.

Mais l'association entre la présence des migrants et une augmentation de la déforestation sur la période correspondante (2000-2007) ne paraît pas facile à établir. En effet, si les secteurs enregistrant une proportion de migrants extérieurs supérieure à $5 \%$ ont enregistré près d'un tiers du déboisement total durant la période, la relation ne paraît pas si claire puisque ces secteurs rassemblent près de $65 \%$ de l'ensemble des migrants, ce qui signifie que les deux autres tiers de la déforestation se réalisent dans des secteurs situés en marge des migrations. D'un autre côté, les 200 secteurs qui ont connu le plus grand déboisement de 2000 à 2007, s'ils représentent environ un tiers du déboisement total sur la période, ne regroupent que 12000 migrants venus d'un autre Etat et 22000 s'étant déplacés dans le même Etat, soit respectivement 7 et $5 \%$ du total des effectifs concernés. Enfin, des analyses de corrélation entre l'importance du déboisement et l'importance des effectifs de migrants (quelle que soit leur provenance), réalisées sur l'ensemble des secteurs ruraux de la zone forestière, ne donnent pas de résultats significatifs. Reflétant l'incertitude de cette relation, pourtant souvent mise en avant, la carte 6 représente les effectifs de population et la proportion de migrants en leur sein, en superposition avec la progression de la déforestation entre 2000 et 2007 dans la région. Comme on le voit, la proportion de migrants est dans la très grande majorité des cas peu importante, même dans les secteurs qui enregistrent une très forte déforestation. Si une relation devait être extrapolée de cette carte, elle pointerait sans doute vers une attraction des migrants non pas exactement dans les secteurs déboisés, mais dans les régions adjacentes déjà un peu consolidées.

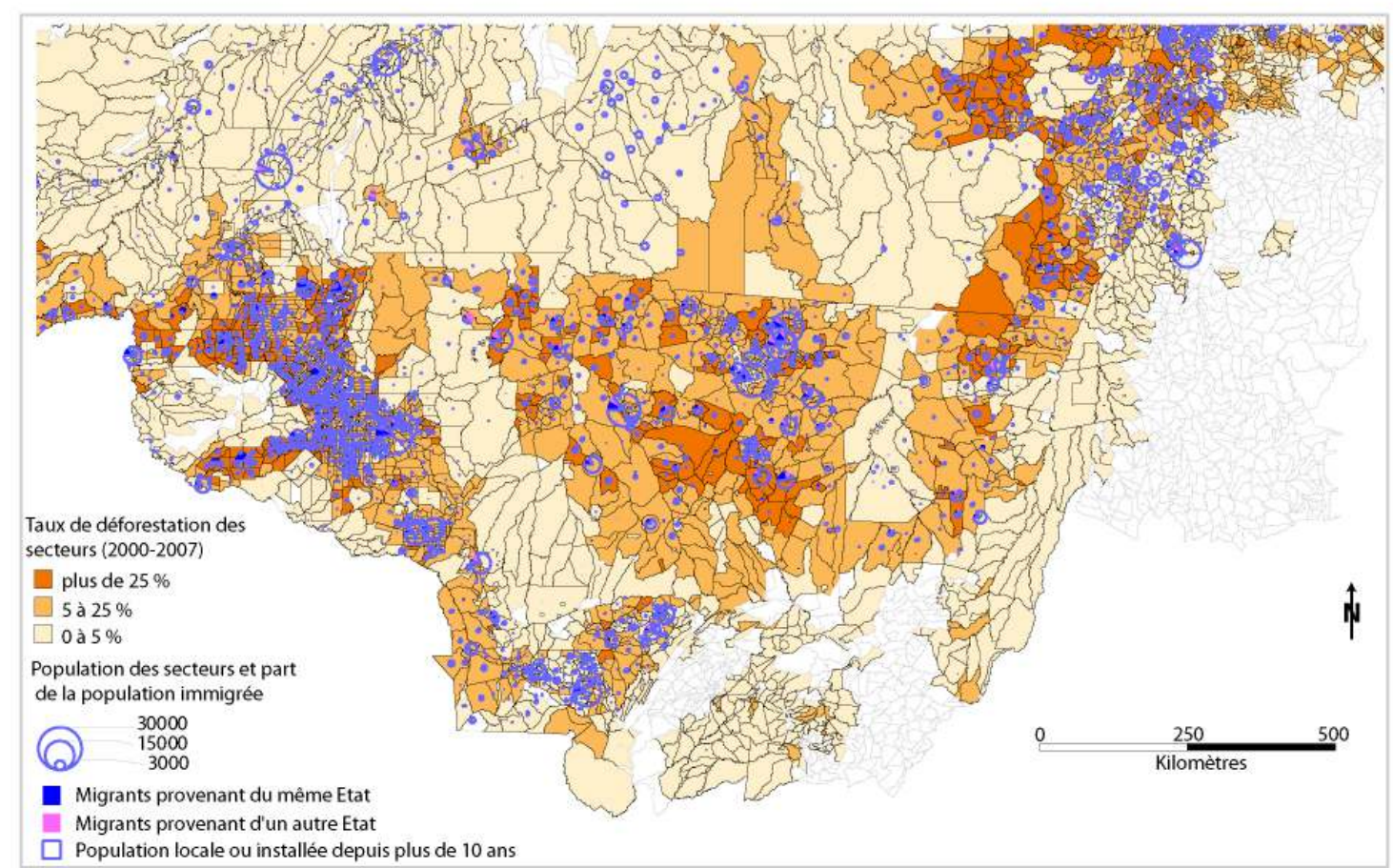

Carte 6: taux de déforestation 2000-2007, effectifs de population et proportion des migrants dans le sud de l'Amazonie.

\footnotetext{
${ }^{14}$ Dont près de 28000 en provenance du Maranhão, soit presque $30 \%$ du total, expliquant l'association entre la présence des Maranhenses et la déforestation dans l'imaginaire de la région.
} 


\section{Des déserts en Amazonie?}

Les secteurs les moins défrichés de l'Amazonie (moins de $5 \%$ de défrichements) représentent 2,856 millions de kilomètres carrés et abritent moins de 1,7 millions d'habitants. Mais cette population est-elle répartie de manière uniforme ? On peut penser que ce n'est pas le cas. Si l'on s'intéresse en effet à la densité de ces secteurs, on s'aperçoit qu'une grande partie des secteurs peu défrichés enregistre une densité de population inférieure à 0,5 habitant par kilomètre carré, mais qu'une autre partie enregistre des densités bien plus élevées.

Nous sommes donc clairement en présence de deux formes d'occupation très différentes. D'un côté les populations traditionnelles, groupées le long des principaux fleuves (voir carte 1), qui occupent relativement densément leur espace (entre 0,5 et 5 habitants par kilomètre carré, voir au delà) sans promouvoir de déforestation. D’un autre côté les espaces « vides » de l'Amazonie, occupés soit par de très faibles effectifs de population traditionnelles, soit par des groupes (non moins faibles en effectifs) amérindiens.

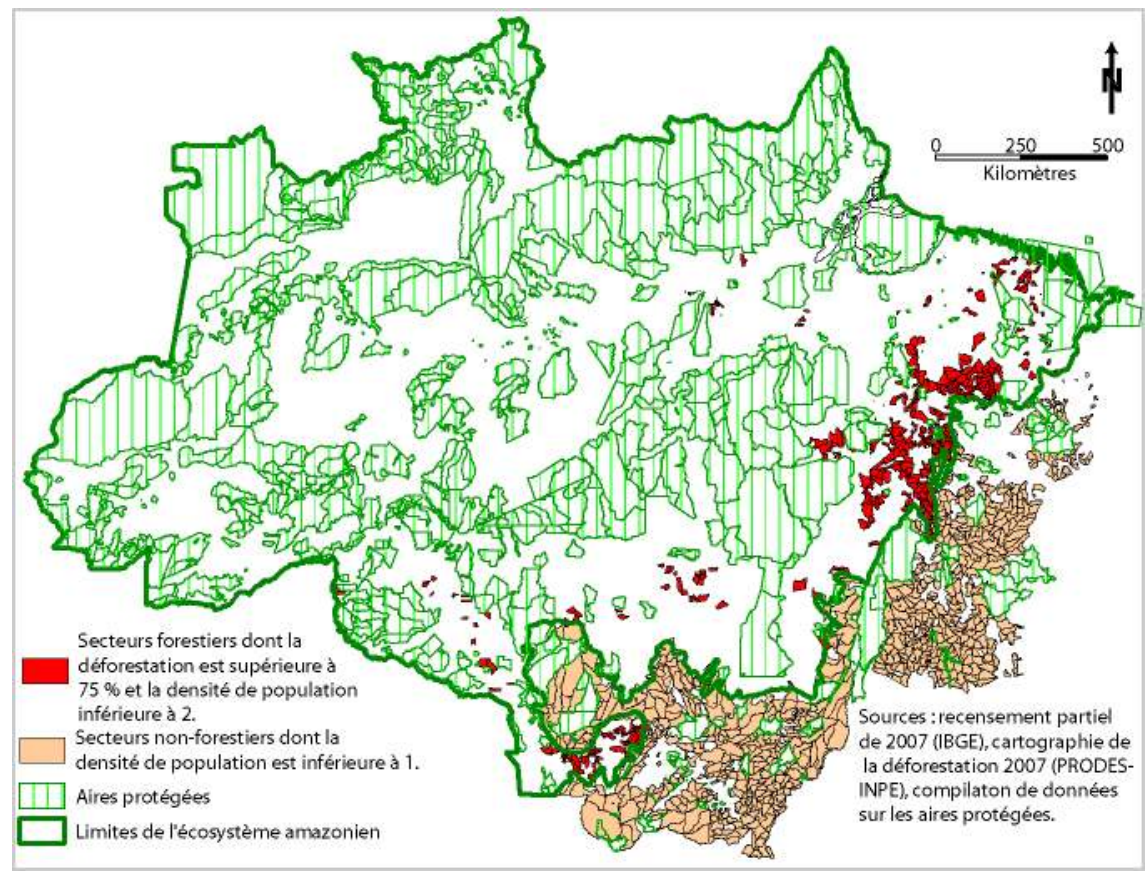

Carte 7 : secteurs défrichés et peu densément peuplés en Amazonie, et secteurs de cerrado de très faible densité démographique ${ }^{15}$.

Au sujet de cette dernière catégorie, on se souvient de la remarque faite en 1925 par le diplomate américain W.L. Schurz: "The Amazon Basin is one of the world's great deserts. An area of 2,000,000 square miles supports a population of less than one to the square mile. It is comparable with the Sahara.". Aujourd'hui cette phrase s'applique encore à un peu moins de la moitié de l'Amazonie forestière, près de 2 millions de $\mathrm{km}^{2} \mathrm{n}$ 'étant occupés que par un peu plus de 120000 personnes, soit une densité moyenne de 0,06 habitants par $\mathrm{km}^{2}$, proche des densités de l'intérieur du Sahara. Cette densité est cependant sans doute légèrement plus élevée, dans la mesure où le recensement sous-estime, comme on l'a dit, les populations amérindiennes les plus isolées, et sans doute également d'autres types de population traditionnelle.

\footnotetext{
${ }^{15}$ Carte établie à partir des données de population du comptage 2007 (IBGE), des données de déforestation de l'INPE et des données de divers organismes sur les aires protégées.
} 
La faible densité de population n'est cependant pas uniquement le fait des zones préservées. On note ainsi que la superficie cumulée des secteurs ruraux dont le défrichement est supérieur à $75 \%$ et dont la densité de population est inférieure à deux représente plus de $104000 \mathrm{~km}^{2}$, n'abritant que ... 102000 habitants (voir Carte 7), soit une densité moyenne inférieure à 1 par $\mathrm{km}^{2}$. Un beau pâturage, en somme, dans lequel la conquête agricole ne semble pas avoir favorisé le peuplement. On notera en passant que ces secteurs ne se situent pas dans des aires protégées (ce qui expliquerait leur faible population), comme le montre clairement la carte 7 .

La même remarque vaut pour le sud de l'Amazonie légale, recouverte originellement par les savanes du cerrado. Bien qu'étant une zone d'agriculture mécanisée à grande échelle (ou peut-être justement du fait de cette caractéristique), la région enregistre une surface d'environ $560000 \mathrm{~km}^{2}$ de secteurs ruraux dont la densité de population est inférieure à 1 et qui se trouvent à l'extérieur des aires protégées (carte 7). Là encore, donc, l'agriculture ne semble pas avoir promu un peuplement très intense des campagnes. Le désert agricole coexiste donc en Amazonie avec le désert forestier. Ces points sont importants dans la mesure où une grande partie du débat sur la question de la protection de l'Amazonie au Brésil se focalisent sur le «vide» des régions amazoniennes ou sur l'importance des surfaces concédées à des groupes amérindiens très peu nombreux (voir Le Tourneau, 2006). Le classique argument «beaucoup de terre, peu d'Indiens » peut donc être facilement retourné en "beaucoup de terre, peu de fermiers $» . .$.

\section{Conclusion}

L'ensemble des remarques qui précèdent permet de poser que l'Amazonie brésilienne, si l'on se base sur la répartition de la population rurale, peut être découpée en quatre zones.

La première est celle de la bordure sud de l'Amazonie. Zone de transition entre les savanes du plateau central et la forêt, elle se caractérise aujourd'hui par une mise en valeur agricole intense, laquelle n'a pas entraîné de peuplement massif, même si les migrants y sont encore importants en proportion de la population.

La seconde région est celle de l'Amazonie défrichée. Elle dessine une large écharpe de l'Acre à la ville de Belém, autrefois appelée "Arc de la déforestation ». Elle possède également une sorte de tentacule s'insinuant au centre de l'Amazonie, le long de la route Transamazonienne. Les densités de population y sont à certains endroits (notamment les zones de colonisation d'Etat) relativement élevées. La région se caractérise également par l'importance de l'élevage extensif dans les activités rurales. Elle est également l'épicentre de l'attraction des migrants internes ou externes à la région, même si leur flux ne semble plus aussi intense que durant les deux décennies précédentes.

La troisième région correspond à l'Amazonie des riverains des grands fleuves (Solimões, Rio Negro, Amazone), appelés ribeirinhos au Brésil. Il s'agit d'une zone dans laquelle les activités traditionnelles sont encore importantes (pêche, collectes diverses) et dans laquelle la densité de population est relativement plus élevée. Le déboisement y est très faible et l'afflux de migrants extérieurs à la région limité à quelques pôles. C'est sans doute une zone dans laquelle des initiatives originales de gestion des ressources naturelles peuvent être recherchées, qu'elles soient traditionnelles ou plus récentes ${ }^{16}$.

La quatrième Amazonie du Brésil est la plus isolée. Elle s'étend sur la rive gauche du grand fleuve, ainsi que dans certaines régions du cours supérieur des fleuves Solimões, Tapajos ou Xingu. Il s'agit de régions très peu peuplées et presque complètement intactes. La

\footnotetext{
${ }^{16}$ Voir par exemple le Projeto Várzea du WWF Brésil ou les initiatives de développement durable de l'Institut Mamirauá. L'analyse d'expériences de développement durable en Amazonie est l'objet du programme DURAMAZ, soutenu par l'ANR (voir http://www.credal.univ-paris3.fr/spip.php?rubrique361)
} 
faiblesse des effectifs de population (ou le fait qu'il s'agit de populations amérindiennes) a fait que cette région a été particulièrement touchée par les politiques de préservation de l'environnement. Elle est aujourd'hui, le siège d'une myriade d'aires protégées qui la recouvrent presque entièrement. Son sort n'est pas scellé pour autant puisqu'une importante de ces aires protégées est dévolue à des populations traditionnelles ou amérindiennes. Le type d'exploitation des ressources qu'elles y développeront et leurs évolutions sociales (Albert et $a l .$, 2008) fera ou non qu'elles demeureront intactes.

\section{Bibliographie :}

- ALBERT B., de ROBERT P., LAQUES A.E. et LE TOURNEAU F.M. (2008) « Aires protégés d'Amazonie brésilienne : territorialité amérindienne et 'Terres Indigènes', continuité ou rupture ? ", in AUBERTIN C. et RODARY E. (eds.), Aires protégées, espaces durables?, IRD, $260 \mathrm{p}$.

- ARNAULD DE SARTRE X. (2006) Fronts pionniers d'Amazonie, Les dynamiques paysannes au Brésil, CNRS éditons, 223 p.

- BECKER B. K. (2004) . Amazônia - Geopolítica na Virada do III Milênio. Rio de Janeiro: Editora Garamond, 172 p.

- BERTRAND J.P., PASQUIS R., APARECIDA DE MELLO N., BOLZON A., CADIER C., GARCIA GASQUES J., LE TOURNEAU F.M., MENDEZ P., PIKETTY M.G., THÉRY H. et WEHRMANN M. (2004) L'analyse des déterminants de l'avancée du front du soja en Amazonie brésilienne : le cas du Mato Grosso, (Rapport sur l'état d'avancement du projet de recherche financé par le Fonds commun INRA-Cirad avec l'appui du Centre de recherche sur le Développement durable (CDS) de l'Université de Brasilia et du CREDAL du CNRS et de l'Université Paris 3), 238 p.

- DELAUNAY, D. (1994), «Fécondités mexicaines: le choix des lieux » in Trace : travaux et recherches dans les Amériques du centre, n²6, pp. 42-61.

- DROULERS M, DEJOUHANET L., SCHWEITZER A.F., SOUCHAUD S., BRUSTLEIN-WANIEZ V., CARRIZO S. et THERY H. (2003), Atlas du Mercosul, publié en ligne sur http://www.iheal.univ-paris3.fr/mercosur_fr/

- DROULERS M. (2004) «L'Amazonie, vers un développement durable, Paris, A. Colin, $228 \mathrm{p}$.

- DUBREUIL V., ARVOR D., MENDEZ DEL VILLAR P., MAGRI FERREIRA C., SIMÕES PENELLO MEIRELLES M. (2007) «Les territoires du soja au Mato Grosso : Développement, crises et adaptation », in Territoires et Sociétés dans les Amériques, 2e colloque du GIS Amérique latine, Rennes

- FEARNSIDE Ph. M. (2003) "Conservation Policy in Brazilian Amazonia: Understanding the Dilemmas", in World Development, vol. $31 \mathrm{n}^{\circ} 5$, pp. 757-779

- GULLISON R.E., FRUMHOFF P.C., CANADELL J.G., FIELD C.B., NEPSTAD D.C., HAYHOE K., AVISSAR R., CURRAN L.M., FRIEDLINGSTEIN, JONES C.D., NOBRE C. (2007) "Tropical forests and climate change" in Science, $\mathrm{n}^{\circ} 316$, pp. 985-986.

- IBGE (2008) Contagem da População 2007 : Agregado por Setores Censitários. Documentação do Arquivo, Ibge:Rio de Janeiro, $42 \mathrm{p}$.

- LAURANCE W.F., COCHRANE M.A., BERGEN S., FEARNSIDE P.M., DELAMONICA P., BARBER C., D'ANGELO S., FERNANDES T., 2001, "The future of the Brazilian Amazon" in Science 291 (5503), pp. 438-439. 
- LE TOURNEAU F.M. (2002) «La représentation du peuplement en pays pionnier : l'Amazonie brésilienne » in L'Espace Géographique, Montpellier, v. 31, n. 2, p. 145152.

- LE TOURNEAU F. M. (2006) «Enjeux et conflits autour des territoires amérindiens en Amazonie brésilienne » in Problèmes d'Amérique Latine, $\mathrm{n}^{\circ} 60$, pp. 71-94

- LE TOURNEAU, F.M. et THERY, H. (2003) «Le Brésil, El Dorado de 1 information géographique ? » in $X Y Z$, v. 94, p. 39-44.

- SOARES-FILHO B.S., NEPSTAD D.C., CURRAN L.M., COUTINHO CERQUEIRA G., ALEXANDRINO GARCIA R., AZEVEDO RAMOS C., VOLL E., MCDONALD A., LEFEBVRE P. et SCHLESINGER P. (2006) "Modelling conservation in the Amazon basin" in Nature, vol 440, n²3, pp. 520-523

- SCHURZ W. L. (1925) "The Distribution of Population in the Amazon Valley" in Geographical Review, Vol. 15, No. 2, pp. 206-225

- THERY H., GONDARD P. et VELUT S. (1997) “Mailles fines pour un grand espace, La carte des divisions statistico-administratives des pays d'Amérique du Sud", Cahiers des Amériques Latines, n²4, IHEAL éditions, pp. 5-37

- THÉRY H., APARECIDA DE MELLO N. (2004) Atlas du Brésil, Paris: La Documentation Française, 302 p.

- VEIGA J. E. (2002) Cidades imaginárias, 2a. ed. Campinas, SP: Editora Autores Associados, $198 \mathrm{p}$.

- VEIGA J. E. (2004), A dimensão rural do Brasil, Document produit dans le cadre des séminaires académiques de la Faculte d'études économiques de l'Université de São Paulo (FEA-USP), disponible sur http://www.econ.fea.usp.br/seminarios/artigos/dimensao_rural.pdf (avril 2009). 